\title{
A model to classify manufacturing archetypes for distributed production
}

\author{
Claudio Sassanelli \\ Department of Economics, Management and Industrial \\ Engineering, \\ Politecnico di Milano, \\ Milan, Italy \\ claudio.sassanelli@polimi.it \\ Claudia Pinna \\ Department of Economics, Management and Industrial \\ Engineering, \\ Politecnico di Milano, \\ Milan, Italy \\ claudia.pinna@polimi.it
}

\author{
Sergio Terzi \\ Department of Economics, Management and Industrial \\ Engineering, \\ Politecnico di Milano, \\ Milan, Italy \\ sergio.terzi@polimi.it
}

\begin{abstract}
Customer demand for more individualization is shifting production models towards decentralized ones. In order to keep up with a heterogeneous class of clients in a turbulent environment, companies are always more inclined towards mass customization. Realizing different kinds of products in line with customer needs without losing the advantages in terms of efficiency of mass production requires new configurations in manufacturing firms as well as a new kind and higher extent of collaboration with customers in the design process. Starting from the analysis of the literature concerning distributed manufacturing, cloud manufacturing and customer involvement, this paper proposes a model aimed at classifying manufacturing archetypes for distributed production.
\end{abstract}

Keywords- distributed manufacturing; cloud manufacturing;customer involvement; archetypes

\section{INTRODUCTION}

In the manufacturing industry, significant changes have been taking place in recent years. Companies are struggling for designing new customer-centric processes and reshaping their supply chains. The notion of distributed production conceptualizes a shift in consumption and production patterns away from conventional mass production, with its long, linear supply chains, economies of scale and centralizing tendencies [1]. This fact is direct consequence of the market becoming a more turbulent environment in which customers are demanding for more personalized goods. Moreover, the growing interdependencies between manufacturing firms are shifting the scope of the design of production systems beyond factory's boundaries. Companies are therefore required to focus on changeability at all levels of their production, from the single station to the network as a whole. Being changeable means to be able to answer to market change with early and foresighted adjustments to the structure and the processes [2]. Furthermore, a key capability for the reaching success in a globalized market is the ability to design, plan and control supply chain scheme that support an efficient collaboration between partners [3]. Besides efficiency, flexible manufacturing technologies are seen also as the main factors enabling mass customization, where customers have stronger presence in the value creation process [4]. The concept of keeping the customer more integrated in the value chain in order to answer more rapidly to his needs switches the attention of manufacturing from a centralized approach to a more distributed paradigm for manufacturing (i.e. distributed production systems). The latter approach has therefore a strong connection with the topic of mass customization. A further opportunity for delivering high variety at affordable prices is indeed coming from the direct response to customer wishes, typically with mini-factories, a new organizational form characterized by the cost reduction potentials achieved through postponement and access to customer sticky information [5]. In most applications of distributed production systems, the customer decides the product that would better fit his needs and he has the possibility to take part in the design and configuration process. The consequence of a more customer-driven market influences the related production processes. Companies are trying to keep up the change and new technologies represent opportunities for developing more suited systems. Distributed systems rely on networks of enterprises, also referred as virtual enterprise networks [6]; hence, the performance of a single company has impact on the overall performance of the network. However, this increasing trend towards collaboration is supporting the rise of cloud manufacturing, which can be considered as a new manufacturing paradigm [7]. This customer-centric and service-oriented model is definitely following the shift from hierarchical business models to more cooperative ones. From this point of view even more groundbreaking concepts, such as social manufacturing, have been proposed. In social manufacturing, production service providers self-organize into dynamic communities to offer manufacturing 
services and collaborate with prosumers [8]. Similarly to cloud manufacturing, other production concepts have been developed after the rise of a certain technology. For example, desktop manufacturing is a concept born after the rapid development of miniaturization technologies [9]. In addition to this, the downsizing of machines is making the deployment of moveable factories feasible, thus improving the quality and sustainability of manufacturing in areas that lack skills or infrastructures [10]. In this context, a model able to classify manufacturing archetypes for distributed production is proposed with this paper. In particular, in par. II the research context is presented, specifying the research questions of this work. In par III the research methodology is described: it is divided in three main blocks and to each of them a par is dedicated. Par. IV presents the literature review, par. V the model definition, par. VI the discussion of its validation. Finally par. VII concludes the paper.

\section{RESEARCH CONTEXT}

It is common knowledge that in recent years the market has been characterized by an increasing pressure for customized products and agile processes. There is a growing consideration for distributed manufacturing systems as a possible answer to these trends. However, neither the proposed decentralized production models nor even the initial reasons of such interest can be considered as uniform. Several frameworks have been proposed starting from studies on decentralized shop floor planning and control, some others derived from the rise of cloud computing or additive manufacturing technologies.

Considering distributed manufacturing as main topic of this work, firstly, given that decentralized production is a wide concept comprising many organizational forms, it is our priority to clarify and classify the main manufacturing archetypes for distributed production. After this preliminary overview, the purpose is to find the main variables to design a classification of the different models. Finally, it is necessary to understand if the interpretative framework previously identified could be used to classify some practical cases related to the topic.

To sum up, the following research questions arise:

- How can we classify the manufacturing archetypes for distributed production?

- Which variables and classification axes could be used to highlight the differences among these archetypes?

There are several papers in literature covering a single aspect (like environmental impact or resilience) of distributed manufacturing, often defining metrics for a comparison with the centralized model. Most of frameworks and models introduced present an in-depth description of single archetypes such as mini-factories or mobile factories. It is pretty common to find journal articles presenting a list of alternatives of decentralized production models, and when this happens, there are no clear variables that help the reader to distinguish among them in a more precise manner. As a source of further confusion, there is a lack of agreement on the terminology to be used to describe such production archetypes. Therefore, the state-of-the-art literature lacks a complete and structured classification model for distributed production archetypes, which could be also used as a bridge between theoretical models and practical cases.

\section{RESEARCH METHODOLOGY}

The methodology used in this work is characterized by three main building blocks (Figure 1): state of the art, modelling and validation. More specifically, the rising relevance of the distributed manufacturing brings directly through the study of the state of the art applying the literature review. The second building block is the core of the work. The maps previously defined are used to elaborate a new map that synthetizes the main concepts about distributed manufacturing. For doing this, we apply a specific methodology called "system thinking", i.e. a way of thinking that makes explicit the relationships and forces that characterize a system. The result is a map giving an overview of specific systems connecting variables with each other. After drafting this framework, it is possible to list a set of relevant variables to be categorized according two dimensions: customer integration and service orientation. At this stage, it is possible to define four main archetypes described by the variables and then they can be positioned in a model having as axes the two dimensions previously identified.

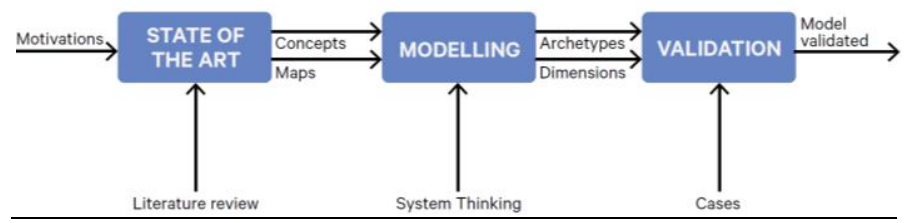

Figure 1 Research Methodology Scheme

The last step consists in finding a way to validate the model, discover its lacks and refine it. This is possible thanks to some real cases describing firms adopting distributed manufacturing. Cases could be, in fact, connected with a specific manufacturing archetype positioned in the model. The model will be validated both in terms of dimensions and archetypes. Firstly, we assess whether the elements and axes chosen are sufficiently able to differentiate cases on the graph. Secondly, we compare their positioning with the one of the theoretical archetypes previously described, in order to highlight potential misalignments between theory and cases.

Finally, the main conclusions on this work are going to be made starting from the results stemming from literature and from the model. Pros and cons regarding the model are run through as well as the possible future developments on distributed manufacturing and of the model itself.

\section{LITERATURE REVIEW}

\section{A. Review process}

Distributed production is a new and complex subject that has been raising interest in both the academic and business world. However, the concept of producing at dispersed locations is more multifaceted than anyone could expect [1]. For this reason, the literature research synthetizes and analyses knowledge coming from diverse sources and theoretical domains. Through this choice, we do not aim to give a systematic and complete summary of a single aspect of the topic, while instead the goal 
is to provide a critical overview of all the most relevant issues of distributed production and its related concepts. In addition to this, a transdisciplinary approach enables management research to gain insights that could not be easily obtained by using knowledge of disciplines separately [11]. In order to analyze the topic, the research process has been structured in three stages (Figure 2): preliminary study, in-depth analysis and selection, organization of the research material.

During the first step, we carried out an initial reading of several scientific articles in order to refine our research idea based on the topic of distributed production. This early effort is also particularly useful for a more considered definition of search keywords and of material selection criteria.

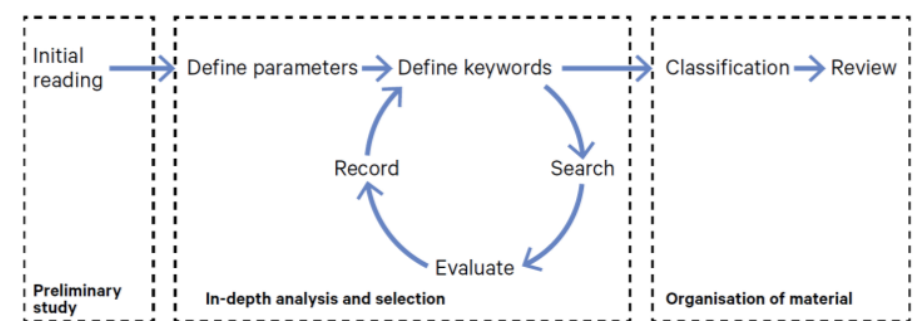

Figure 2 Literature search and review process

In the second step, the search and filtering of papers actually took place. In terms of parameters, the search strategy was little constrained. There were no restrictions in terms of subject area, geographical area or publication period. Indeed, as said before, the topic became relevant only in recent years and it addresses various disciplines. Much attention has been paid to the type of literature, including in the survey only reports, conference proceedings and research papers. Three approaches have been applied for the collection of these sources: searching on academic search engines, obtaining relevant material referenced in articles already read and searching through Internet. To be precise, literature search was made mainly thanks to Web of Science and Scopus databases. In doing this, a set of keywords, updated as long as the literature analysis progressed, to filter the academic database was used. Indeed, during the second stage an iterative process consisting in keyword definition, search, selection and recording was followed: each iteration corresponded to a more precise approach to the subject. The selection of relevant articles, besides keywords analysis, was undertaken by carefully revising if the title was coherent with the main topics treated. Then, more in-depth evaluation was characterized by the reading of the abstracts (or the entire content when needed).

In this section, a critical review of the literature related to distributed production is also presented. As it is possible to notice, the topics of distributed manufacturing and cloud manufacturing have something in common. Both are pushing towards the decentralization of production into dispersed factories but the way they are doing this is different. Indeed, research about distributed manufacturing comes from studies on production planning and control, while cloud manufacturing is a much more recent topic emerging from the advent of cloud computing. In spite of the two quite separate lines of research, there is a small intersection represented by papers which are grouping distributed manufacturing archetypes and cloud production in a single set of production models. Indeed, while cloud manufacturing literature can be easily structured into key concepts, architectures and value drivers, the literature related to distributed manufacturing is too diversified to be constrained into these rigid components, leading to detect some major topics such as collaborative networks, organization philosophies, frameworks, mini-factories and mobile ones. A third line of research is related to the role of the customer: only the aspects meaningful for the topic of distributed production were taken into account. Indeed, the literature about desktop manufacturing evolved in connection with several concepts related to the empowered role of the customer.

In the following the concepts of distributed manufacturing, cloud manufacturing and customer involvement are introduced.

\section{B. Distributed Manufacturing}

In recent years, the term Distributed Manufacturing has been getting more and more attention in research projects related to new manufacturing paradigms. Despite its highly relevant nature for current competitive challenges in the manufacturing sector, the concept is not new and it has taken on different connotations over the years. The first interpretation is related to decentralized production control. In this first stage of evolution, researchers introduced the concept of Distributed Manufacturing Systems (DMS) as a class of manufacturing systems characterized by an innovative architecture focused on decentralized decision making within the factory. According to [12], this class of manufacturing systems shows common properties such autonomy, flexibility, adaptability, agility, decentralization. Therefore, compared to the general concept of Distributed Manufacturing, DMS refer to independent manufacturing units formed within the shop floor where certain control powers are distributed to each of these units. The second interpretation of DM term is related to the concept of creating value at geographically dispersed locations. In other words, in this case the company decentralizes its production system at more than one factory. Finally, a third evolution of the term crossed the boundaries of the single enterprise, settling in the context of production network. Indeed, the term "Production Network" refers to the cases when there is a manufacturing cooperation between at least two legally independent enterprises. In this third stage, the term of Distributed Manufacturing Network is strongly related to the concept of Collaborative Networked Organization (CNO) presented by [6].

\section{Cloud Manufacturing}

Cloud Manufacturing (CM) is an emerging manufacturing paradigm strongly related with the concept of cloud computing. According to NIST (National Institute of Standards and Technology) [13], cloud computing is defined as "a model for enabling ubiquitous, convenient, on-demand network access to a shared pool of configurable computing resources (e.g., networks, servers, storage, applications, and services) that can be rapidly provisioned and released with minimal management effort or service provider interaction". Based on the work of 
NIST and Smith, Wu proposed the following definition: "Cloud Manufacturing (CM) is a customer-centric manufacturing model that exploits on-demand access to a shared collection of diversified and distributed manufacturing resources to form temporary, reconfigurable production lines which enhance efficiency, reduce product lifecycle costs, and allow for optimal resource loading in response to variable-demand customer generated tasking".

$\mathrm{Wu}$ [14] describes also a tri-group model, called providerconsumer interaction model, based on the interaction of three different layers. The first layer relates to users, which are individuals or groups that want to gain a competitive advantage by utilizing the cloud. They could be individuals who do not possess capabilities to produce or even companies which find more convenient to outsource production to $\mathrm{CM}$. The second layer of the model is represented by application providers, which are responsible for managing the cloud environment and for translating user requirements into the data required for the production. The third layer is operated by physical resource providers (PRPs), those who own the manufacturing equipment and the necessary expertise to operate it efficiently and effectively. It is important to highlight that PRPs join the cloud manufacturing network in relation to their know-how and experience, rather than to their geographical location. As underlined by the definition, customer-centricity is one of the key characteristics of Cloud Manufacturing. Traditional supply chains, based on the hierarchical flow of requirements from suppliers to sub-tier suppliers, are too rigid for exploiting completely the opportunity represented by the enriched customer experience. The key aspect for $\mathrm{CM}$ is the ability to match users, with their specific needs, to resource providers who can satisfy those requirements while meeting cost, schedule and quality objectives. The resulting benefits are enhanced efficiency, reduced cost, increased flexibility and improved capabilities for the user. However, these benefits are actually achievable thanks to the pooling of PRPs resources which enable the creation of flexible manufacturing sequences. CM production lines are designed to be temporary, quickly reconfigurable and dynamic. The property of system flexibility is however related to the use of automation and Industrial Control Systems (ICS), able to control and support the flow of production requirements from the cloud to multiple dispersed manufacturing facilities with a minimized down-time. The resulting benefits are high efficiency, low down-time and instant response to demand. In addition, the quick re-configurability of the system has an important side effect because also production in small lots becomes economically viable. Ideally, the CM environment would be able to process even any job, without disruption of larger ones. Moreover, the CM environment is intended to be demand intelligent, meaning that the system is flexible enough to provide load sharing across interchangeable resources. Finally, the last characteristic of CM is related to the possible business models. Indeed, business innovation could come also from the adoption of a share-to-gain philosophy. In particular, one of the key challenges is the decision on how the value of the final products should be divided across contributors.
With an extremely dynamic cloud of resource providers, there is not in fact a clear and stable representation of how value is added, which is given by value chain structure. Instead, new business models would be needed to deal with new issues, such as the role of app providers and the ownership of intellectual property.

\section{Customer involvement}

As mentioned in the description of network of enterprises, customer satisfaction is the ultimate scope. In fact, the client is being involved at a higher extent in the value creation process. In order to keep the pace with market changes and adapt to customer taste, companies associate with each other to achieve mass customization [15]. In fact, mass customization is a strategy aimed at satisfying customer needs with price typical for mass production [16]. Hence, MC enables the opportunity for companies to design, manufacture and provide customers with large quantities of different products tailored to specific customer needs in the same range of price and time applied by mass manufacturers [17]. In this context, the enterprise has to manage the variability of demand coming from different consumer's needs, transform the needs into customized products and then to arrange the best manufacturing process to realize them in an efficient way. In the current turbulent environment firms try to build collaborative networks to cope with these challenges. This form of relationship implies "sharing risks, resources, responsibilities and rewards" [15].

\section{E. Literature review results}

The presentation of the general map and the discussion of gaps in literature are concluding the review. The map (Figure 3 ) is the result of the analysis stemming from literature. The approach used to derive it was bottom-up; from the specific archetype maps a new one was obtained, synthetizing the other ones. In other words, the result is a summary of the other maps achieving a lower level of complexity. Value drivers are underlined in red colour, elementary variables in green, while link variables are in grey. The relevance of each link in the map was deducted from the number of authors discussing the topic and from the specific importance given by them in the papers. The map takes into account the main variables able to synthetize the main concepts regarding distributed manufacturing. In addition to this, similar concepts were also grouped in order to avoid redundancy and, consequently, reducing the degree of complexity and enhancing clarity of the map.

To sum up, the main conclusions deriving from these steps were:

1) Disagreement and contrast in the terminology of the topics in literature used by different authors. In fact, many concepts are used as synonyms in some cases while in others as completely different ones. An example is the concept of desktop factories. After reviewing literature on the topic, we chose this terminology as the umbrella term for several concepts (micro-factories, open production, fablab, etc). In other cases, the term micro-factory is used as a synonym of mini-factory, which is instead a pretty specific model in literature. 
2) Misalignment in the link between real cases and literature. In the description of the cases many current terms differ from the same in the literature. This is especially true for example in the context of cloud manufacturing. It is possible to see that some cases outlined in literature (e.g. Quirky) are cited as an example of both cloud and social manufacturing. However, the misalignment arises when the same authors define cloud and social manufacturing as distinct production models. What we have concluded after the review of such topics is that social manufacturing can be considered as a specific development of the cloud paradigm. Therefore, given that the two concepts are neither completely overlapping nor completely distinct, the issue of assigning real-world cases to their appropriate concept still remains.

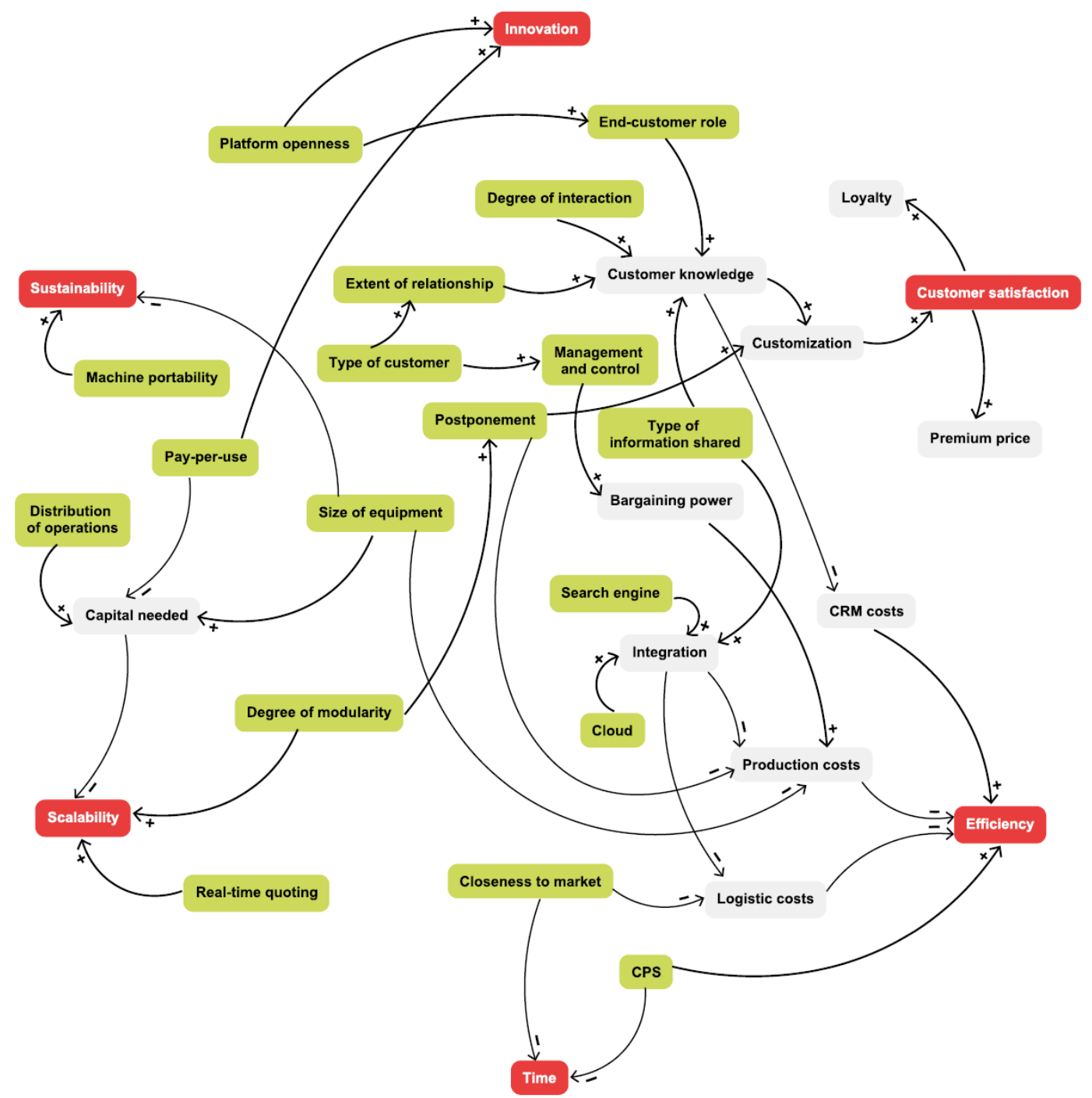

Figure 3 General causal loop diagram

\section{MODELLING}

After the literature review, the main variables identified were used to build the model able to classify the different archetypes concerning distributed manufacturing. In this section the model is described in all its components. For the development of this model we started from what emerged in the literature review. In particular, the careful reading and analysis of articles allowed to detect the essential relationships between concepts and topics. Moreover, moving beyond the features of the specific production model presented (i.e. the archetypes), those dynamics have been generalized in a single map with the help of system thinking methodology. In this way, it has been possible to build the chain of relationships that links the basic elements of a production system to its broad value drivers. Being reassured by the fact that those relationships are founded in peer-reviewed articles, the nodes and links of the general map were used as the starting point of the model. The nodes of the general map belong to different levels. Therefore, as shown in Figure 4 the building blocks of the model are organized in 
accordance with the different levels. The chart lists four different levels: elements, axes, archetypes and value drivers.

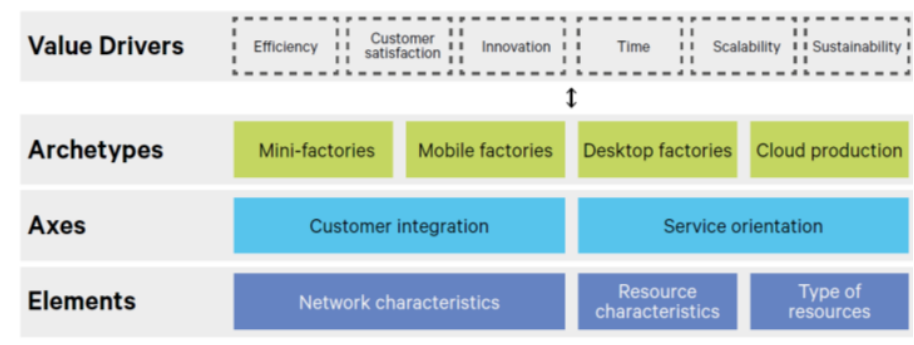

Figure 4 Building blocks of the model

The elements (Figure 5) are the fundamental components of a production system model. They have been defined in such a way that they would be able to capture how different production networks are operating. Elements could be of three types: network characteristics, resource characteristics and types of resources.

\begin{tabular}{|l|l|l|}
\hline $\begin{array}{l}\text { Network } \\
\text { characteristics }\end{array}$ & $\begin{array}{l}\text { Resource } \\
\text { characteristics }\end{array}$ & $\begin{array}{l}\text { Type of } \\
\text { resources }\end{array}$ \\
\hline \hline End-customer roles & Closeness to market & Cloud \\
\hline $\begin{array}{l}\text { Type of } \\
\text { customer }\end{array}$ & Distribution of operations & CPS \\
\hline Degree of interaction & Degree of modularity & Search engine \\
\hline Extent of relationship & Size of equipment & $\begin{array}{l}\text { Real-time quoting } \\
\text { system }\end{array}$ \\
\hline Point of postponement & Machine portability & Pay-per-use model \\
\hline Management and control & Information shared & \\
\hline Platform openness & \multicolumn{2}{|l}{} \\
\cline { 1 - 2 } & &
\end{tabular}

Figure 5 Elements of the model

The upper blocks of the model consist in the classificatory axes. The customer integration and the service orientation represent the two main dimensions used to classify a production model.

The first classificatory axe chosen for the model is customer integration. The level of customer integration is defined by the extent to which a customer takes part to value-creating processes. Thus, it could be seen as a measure of how much customer roles extend to the scope of activities traditionally associated to companies. In the context of customer integration, there is room for several trending concepts such as co-creation, presumption and crowdsourcing. Of all these topics, customer integration was chosen as a main axe because it is a concept able to address most of the elements previously described. In addition to this, it is an easily understandable concept able to distinguish between different production archetypes.

To complete the model, it is necessary to find a second dimension able to classify production archetypes, the service orientation. Indeed, while network elements contribute to determine the level of customer integration, the dimension defined by the resources used and their properties is quite subtle. The set of resources considered include both pure information technologies, such as social media, and technologies that operate on the border between digital and asset worlds. The characteristics of resources included in the model are instead showing the fragmentation of activities and location specificity as possible interesting dimensions. In order to combine all these aspects in an overall view, we decided to rely on the level of service orientation in production as our second classificatory axe. We define therefore the level of service-orientation as the extent to which manufacturing resources and process are delivered to the network in the form of services.

At an even higher level, we found archetypes, with the related demarcated areas on the bi-dimensional space defined by the classificatory axes. Those areas correspond to the four main production model archetypes found in literature.

Mini-factories are a specific form of new organizational structure required for mass customization. These structures are taking the form of integrated sales, innovation, customer interaction and production units that are in close proximity to the customer [5]. The typical customer of a mini-factory is a simple consumer, asking for customized products and/or services.

A different production archetype is represented by mobile factories. The term refers to moveable production facilities that could be easily deployed at different locations. There are three possible types of mobile factories: individual factories housed in large vans when location changes weekly, sets of several container size factory units when location changes monthly or yearly, modular factories with pre-fabricated elements for more stable locations [10]. Even if processes need to be adapted to local conditions, the possibilities for the consumer to customize the goods is quite limited.

Another key production archetype consists in desktop factories. The concept has been developed as a consequence of several trends such as the downsizing of production equipment and their democratization, the involvement of customers in the role of prosumers, the use of additive technologies that enable singlebatch production. There are three scenarios for desktop factories: manufacturing at the point of use, manufacturing in neighborhood facilities and home fabrication. The desktop factory is the archetypes with the highest level of customer integration.

Cloud manufacturing is a customer-centric manufacturing model that exploits on-demand access to a shared collection of di- versified and distributed manufacturing resources to form temporary, reconfigurable production lines which enhance efficiency, reduce product lifecycle costs, and allow for optimal resource loading in response to variable-demand customer generated tasking [14]. Differently from the previously described models, cloud production offers a great flexibility in its implementation. Depending on who is joining the cloud, the network can configure itself to accept different levels of customer integration. 
Once described the four archetypes in terms of the model elements, the next step is to place them in the bi-dimensional space created by the two axes. Figure 6 shows the positioning of the production archetypes.

Lastly, the highest level of the model is dedicated to value drivers, which are basically a rationalization of all the factors leading a production network to create value for a company.

Furthermore, these building blocks are stacked up because the analysis performed at each level is necessarily derived from information contained in the lower one. Finally, as final step, the proposed model has been validated through its application on eleven real cases.

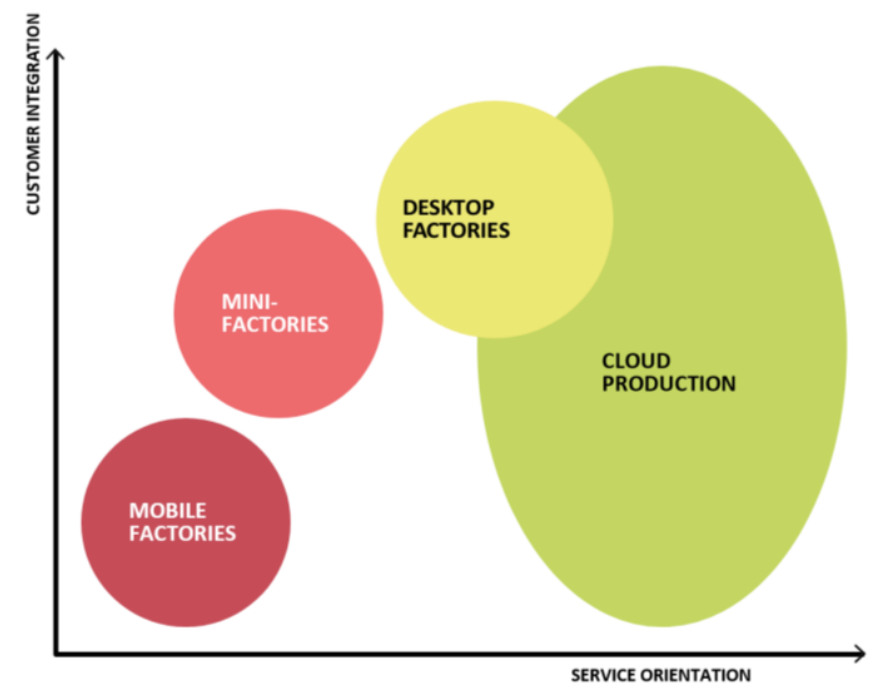

Figure 6 Positioning of the four production archetypes

\section{VALIDATION AND DISCUSSION}

So far, the model has been drafted following a deductive approach, which started from the identification of the main production concepts and most relevant variables in the literature. To validate and further refine this work, some applications with real world cases ware performed: this allowed the authors to understand the accuracy of the model and improve it. First of all, the goal was to test the model ability to classify cases properly. Therefore, each case was put on the bidimensional chart according to the value of their variables. Given that the selected cases are quite different, they were supposed to be spread all over the chart: this would have confirmed that the model is able to classify them sufficiently. The second aim of the validation phase is to check whether theoretical production archetypes are matched by clusters of cases emerging from the real world. Archetypes could be seen as selective representations of reality, therefore it is possible that they are not fitting with real-world cases.

From Figure 7 it is possible to detect three different clusters. The first on the left includes Facit Homes and Local Motors Mobifactory. This group has approximately the same positioning of the mobile factory production archetypes. Therefore, it is possible to say that the theoretical model of

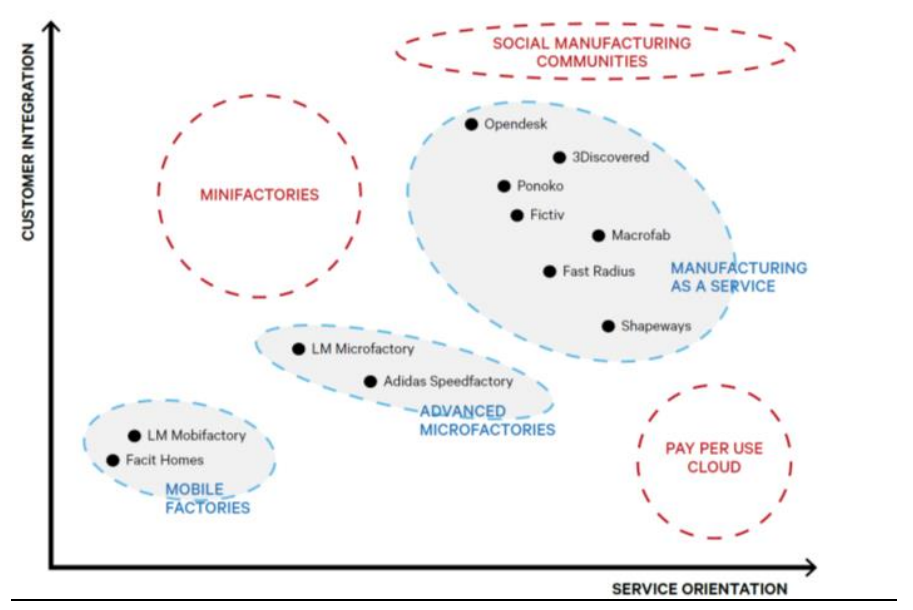

Figure 7 Cases positioned on the chart

mobile factories is validated by the presence of these two practical applications. With regard to the second cluster, which we called advanced microfactories, there is a slight misalignment between theory and real-world cases.

Moreover, they show features that are more typical of a desktop factory, such as the extensive implementation of direct digital manufacturing. However, compared to the scenarios described for desktop factories, this group of cases appears to be closer to the industrial context. Indeed, the concept of desktop factories encompasses also non-industrial scenarios, such as home fabrication or manufacturing-at-the mall, where customization happens directly in the retail store. In both cases, one critical aspect of the design of desktop factories lies in the ability to maintain safety and ergonomics even in a more domestic environment. On the contrary, what we call advanced microfactory includes technologies like cyber-physical systems, ambient intelligence, sensors and advanced automation, so far confined to the industry level.

Advanced microfactories are also not completely similar to mini-factories. While customer knowledge is definitely relevant as in mini-factories, the level of customer integration is slightly lower. In addition to this, automation is used to a greater extent, allowing these production systems to be much more efficient than mini-factories. The area dedicated to mini-factories is therefore empty. Given that there could be small and little known applications of mini-factories, there could be another possible explanation of this lack. The theoretical model of minifactories has been developed more than a decade ago, when opportunities represented by cyber-physical systems, increased automation and 3D printing were totally unforeseen. Hence, it is quite reasonable that the mini-factory concept is not exactly matched by practical cases, especially if we consider that it is a clearly delimited model in terms of technologies. From this perspective in fact, the previously-mentioned opportunities are a necessary development to better serve the purpose of mass customization. Moving to cloud production, it is possible to see that the practical cases are situated in the middle-left of the area covered by the archetype. As a consequence, we could say that reality confirms the variety of applications of cloud production. However, in the field of cloud production there are two 
uncovered areas that need further explanation. The first, on the upper right, is the space related to social manufacturing communities. In this area, we would have expected to find the most advanced applications of the cloud paradigm. Among these, we include communities of production providers who self-organize themselves to provide manufacturing services. According to this scenario, the management of the cloud would be partially decentralized and there would be an even greater involvement of the customer. None of the cases analyzed seems to follow the requirement of self-organization. In addition to this, even the cases presented in literature appear to be more similar to the cases analyzed than to the concept of social manufacturing. We could explain the existence of this gap by considering the social application as a further development of the centralized platforms for cloud manufacturing. From this perspective, it is reasonable to say that it is unlikely that groups of producers realize the change and self-organize themselves into communities without being mediated by a platform. Hence, this kind of platforms represents the most realistic implementation of the cloud paradigm at the moment. On the bottom-right, another advanced application of cloud seems to lack. This space should be occupied by platforms that orchestrate the exchange of manufacturing services according to a pay-per-use model. Platform users should pay for resource consumption, rather than for a product. This scenario, very common in the field of cloud computing, is not matched by any of the cases analysed. As a possible explanation, we should consider that it is the most technologically demanding application, because it requires a very high level of service orientation of the system. For example, in order to enable a payper-use business model, each machine needs sensors for monitoring in real-time the consumption of resources. In addition, the modularity of services should be even higher, in order to allow the user to pay exactly for the activities actually performed. However, in the cases analyzed the coordination of production providers at the network level involves only the exchange of design files, the allocation of orders to different producers and, optionally, the scheduling of production activities. It is therefore reasonable to say that the current amount of information shared in the network is not sufficiently detailed to allow an effective pay-per-use business system.

\section{CONCLUSIONS}

The presented work focused its attention on a new paradigm of production in contrast with the mainstream concept of centralized production, i.e. distributed manufacturing. What emerges from the context analyzed is that customer demand for more individualization is shifting production models towards decentralized ones. In order to keep up with a heterogeneous class of clients in a turbulent environment, companies are always more inclined towards mass customization. Realizing different kinds of products in line with customer needs without losing the advantages in terms of efficiency of mass production requires new configurations in manufacturing firms as well as a new kind and higher extent of collaboration with customers in the design process. During the analysis of literature, we found that the area regarding distributed production was extremely wide including different models, value drivers and main trends descriptions. Hence, it was necessary to give order to literature themes and relating them with the main topic of distributed production. Literature lacked a comprehensive reordering and classification of the main archetypes of distributed manufacturing. Consequently, after distinguishing among the archetypes, a model for highlighting the main characteristics and differences among them has been proposed. The axes that we identified as the main dimensions were customer integration and service orientation. The former was introduced considering the rising trend of customer involvement in value creation process. The latter was related to the bias towards the delivery of a product or a service. According to the model, it was possible to have a clear distinction of the archetypes; this kind of classification was not still present in literature as well as a proper reordering of the main factors affecting the concept of distributed manufacturing (final map). What is clear is that distributed production is more just than a little fad and more and more companies are likely to enter the arena if performances and profits will demonstrate to be consistent. Furthermore, it was necessary to look for some practical cases in order to see if the model was robust in real contexts. First of all, it was clear that terminology used by "cases writers" was not as specific as the classification of the archetypes of the model proposed. In fact, all cases found were referred to as distributed manufacturing in general terms. Customer integration and service orientation were extrapolated by means of deduction from firm corporate sites and secondary sources. The cases put on the model confirmed that four main clusters exist. However, there is a misalignment between literature and real cases grouping. Although the process followed for the model realization was quite structured, pros and cons related to the model can be detected in Table 1 .

\section{Table 1 Pros and cons of the model}

\begin{tabular}{|l|l|}
\hline PROS & CONS \\
\hline 1) Area of the graph is covered in \\
largest part: The model highlights \\
the presence of four main clusters \\
covering the largest part of the \\
space, showing that the variables \\
from the model: Although the \\
variables selected were stemming \\
from literature analysis and research \\
on scientific papers, we cannot be \\
can keep them well separated from \\
each other. In this way, the model \\
could be quite exhaustive and \\
thecise to bring focus to the \\
variables excluded from the analysis \\
that could make the separation of \\
the archetypes more evident in the \\
model.
\end{tabular}


this, the number of variables is to numbers attributed to variables sufficient enough to guarantee the identified.

robustness of the model derived.

Moreover, according to the model developed in this work, there are some aspects concerning distributed production that could be carefully taken into account by firm managers.

First of all, there are firms, as seen in the validation cases, that apply distributed manufacturing even though they have not proper understanding of which kind of distributed production they are working on. The archetypes found and the position on the model can give a comprehensive overview of the main structures present in this context. In this way, managers can realize where the firm could be positioned and have a more in depth understanding of which variables are connected and how they can influence the associated value drivers. Given the simplicity of the model comprising only two main dimensions, this operation of a self-collocation in the model by a firm as well as a clearer understanding of the positioning of other firms could highlight the main differences in the competitive arena. Secondly, firms that are external from the context but that could perceive the necessity of an evolution towards distributed production in the future could have more awareness of the possible scenarios and of the main variables that characterize new configurations. For example, if a firm is willing to change its overall strategy focusing on distributed production, it could opt for a configuration similar to one of the archetypes of the model. If it wants to act as an intermediary between demand and supply, configurations more biased towards cloud production could be more effective, considering that the provision of a service is prerogative. On the contrary, if a firm wants to be more focused on the realization of a product with a low level of interaction with the customer in the design process, mobile factory configuration could be more appropriate.

According to the results and performances achieved by firms leveraging on distributed manufacturing, other firms could take more aware decisions on which characteristics to focus the attention, be competitive in the arena but also they could understand the key success factors for winning in the market. Nevertheless, the presented model has not the purpose to tell managers which is the most convenient archetype of distributed manufacturing. In fact, a quantitative study of the variables in the cases as well as a connection among them and the performances was not executed. Hence, a possible evolution of the study could cover this area following a more quantitative approach and trying to link variables and performances of a relevant number of cases. Finally, a further point for future researches could be to take in account also the context of platform economy in the study, since some of the developments in this field appear to come close to cloud manufacturing ideas, practically leading to the birth of manufacturing hubs and marketplaces for manufacturing capacity.

\section{REFERENCES}

C. Kohtala, "Addressing sustainability in research on distributed production: An integrated literature review," J. Clean. Prod., vol.
106, pp. 654-668, 2015.

H. P. Wiendahl et al., "Changeable Manufacturing - Classification, Design and Operation," CIRP Ann. - Manuf. Technol., vol. 56, no. 2, pp. 783-809, 2007.

[3] D. Mourtzis, M. Doukas, and F. Psarommatis, "Design and planning of decentralised production networks under high product variety demand," Procedia CIRP, vol. 3, no. 1, pp. 293-298, 2012.

F. T. Piller, K. Moeslein, and C. M. Stotko, "Does mass customization pay? An economic approach to evaluate customer integration," Prod. Plan. Control, vol. 15, no. 4, pp. 435-444, 2004.

R. Reichwald, C. M. Stotko, and F. T. Piller, "Distributed minifactory networks as a form of real-time enterprise: Concept, flexibility potential and case studies," Pract. Real-Time Enterp. Facts Perspect., pp. 403-434, 2005.

[6] L. M. Camarinha-Matos, "Collaborative networked organizations in manufacturing," IFAC Proc. Vol., vol. 1, no. PART 1, pp. 187-198, 2007

[7] D. Wu, D. W. Rosen, L. Wang, and D. Schaefer, “Cloud-based design and manufacturing: A new paradigm in digital manufacturing and design innovation," CAD Comput. Aided Des., vol. 59, pp. 1-14, 2015.

[8] P. Jiang, K. Ding, and J. Leng, "Towards a cyber-physical-socialconnected and service-oriented manufacturing paradigm: Social Manufacturing," Manuf. Lett., vol. 7, pp. 15-21, 2016.

[9] R. E. DeVor, J. Cao, K. F. Ehmann, and S. G. Kapoor, "Transforming the Landscape of Manufacturing: Distributed Manufacturing Based on Desktop Manufacturing (DM)2," J. Manuf. Sci. Eng., vol. 134, no. 4, pp. 041004-041004, 2012.

[10] S. Fox, "Moveable factories: How to enable sustainable widespread manufacturing by local people in regions without manufacturing skills and infrastructure," Technol. Soc., vol. 42, pp. 49-60, 2015.

[11] M. Saunders, P. Lewis, and A. Thornhill, Research methods for business students. 2009

[12] P. Sousa, N. Silva, T. Heikkila, M. Kollingbaum, and P. Valckenaers, "Aspects of Co-operation in Distributed Manufacturing Systems," in Proceedings of the 2nd International Workshop on Intelligent Manufacturing Systems, 2001, pp. 1-21.

[13] P. M. Mell and T. Grance, "The NIST definition of cloud computing," Gaithersburg, MD, 2011.

[14] D. Wu, M. J. Greer, D. W. Rosen, and D. Schaefer, "Cloud manufacturing: Strategic vision and state-of-the-art," J. Manuf. Syst., vol. 32, no. 4, pp. 564-579, 2013.

[15] A. Giovannini, A. Aubry, H. Panetto, and H. El Haouzi, "Mass customisation in sustainable networked enterprises," IFIP Adv. Inf. Commun. Technol., vol. 408, pp. 670-678, 2013.

[16] M. Walczak, "Models of the Emergence and Diffusion of Mass Customization," Procedia - Soc. Behav. Sci., vol. 110, pp. 812-821, Jan. 2014.

[17] Q. Tu, M. A. Vonderembse, and T. S. Ragu-Nathan, "Manufacturing practices: antecedents to mass customization," Prod. Plan. Control, vol. 15 , no. 4, pp. 373-380, Jun. 2004. 